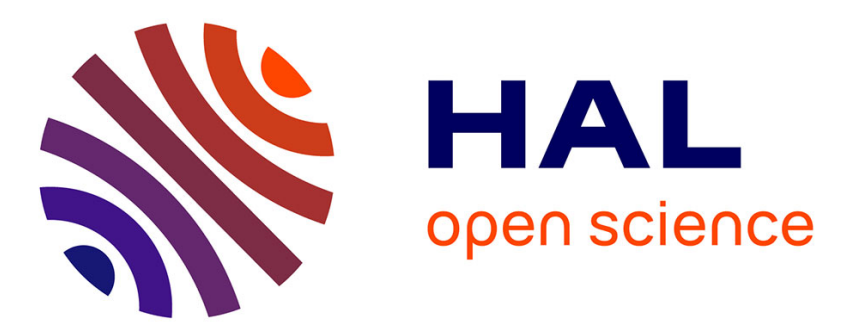

\title{
Une petite nécropole du début de La Tène moyenne à Mory-Montcrux (Oise) \\ Jean-Claude Blanchet
}

\section{To cite this version:}

Jean-Claude Blanchet. Une petite nécropole du début de La Tène moyenne à Mory-Montcrux (Oise). Revue archéologique de Picardie, 1983, Les Celtes dans le nord du bassin parisien. Actes du Ve colloque de l'Association française pour l'étude de l'âge du Fer, 1, pp.66-73. 10.3406/pica.1983.2987 . hal-02535673

\section{HAL Id: hal-02535673 \\ https://hal.science/hal-02535673}

Submitted on 7 Apr 2020

HAL is a multi-disciplinary open access archive for the deposit and dissemination of scientific research documents, whether they are published or not. The documents may come from teaching and research institutions in France or abroad, or from public or private research centers.
L'archive ouverte pluridisciplinaire HAL, est destinée au dépôt et à la diffusion de documents scientifiques de niveau recherche, publiés ou non, émanant des établissements d'enseignement et de recherche français ou étrangers, des laboratoires publics ou privés.

\section{(ㅇ)(1) $\$$}

Distributed under a Creative Commons Attribution - NonCommercial - NoDerivatives| 4.0 


\title{
UNE PETITE NECROPOLE DU DEBUT DE LA TENE MOYENNE A MORY-MONTCRUX (OISE)
}

\author{
par Jean-Claude BLANCHET *
}

\section{CIRCONSTANCES DE L'INTERVENTION}

Nous avons déjà eu l'occasion de présenter dans une note préliminaire cette petite nécropole gauloise, après la fouille de sauvetage menée du 13 Juillet jusqu'à la fin du mois d'août 1974 (BLANCHET J.-Cl. et JACQUINEZ R., 1975).

Cette découverte remonte au mois de Juin 1974 lorsque nous avons été averti de la mise au jour d'une poterie quasi intacte dans une carrière de craie ouverte à environ un kilomètre au nord-est de Mory-Montcrux (Oise). C'est un agriculteur du village, Monsieur MAILLARD, qui trouva l'objet en extrayant de la craie pour remblayer ses chemins et sa cour de ferme. II nous emmena sur les lieux où nous avons pu recueillir les fragments d'une coupe à ombilic dans les éboulis de la paroi nord de la carrière. Il ne restait plus de trace d'une éventuelle sépulture. Monsieur MAILLARD nous indiqua qu'il n'avait jamais rien remarqué auparavant (1). Devant l'extension menaçante et non régulière de la carrière, le Service des Antiquités Historiques de Picardie nous demanda d'opérer un intervention de sauvetage. C'est une bande de 12 mètres de longueur en front de carrière et de 8 mètres de largeur qui fut entièrement fouillée. Par la suite, nous avons ouvert des sondages à l'intérieur de la friche pour vérifier l'extension possible de la nécropole. Aucune autre sépulture ne fut rencontrée.

\section{LE MILIEU NATUREL}

Mory-Montcrux est une petite commune du département de I'Oise (canton de Breteuil-sur-Noye - arrondissement de Clermont voir fig. 1).

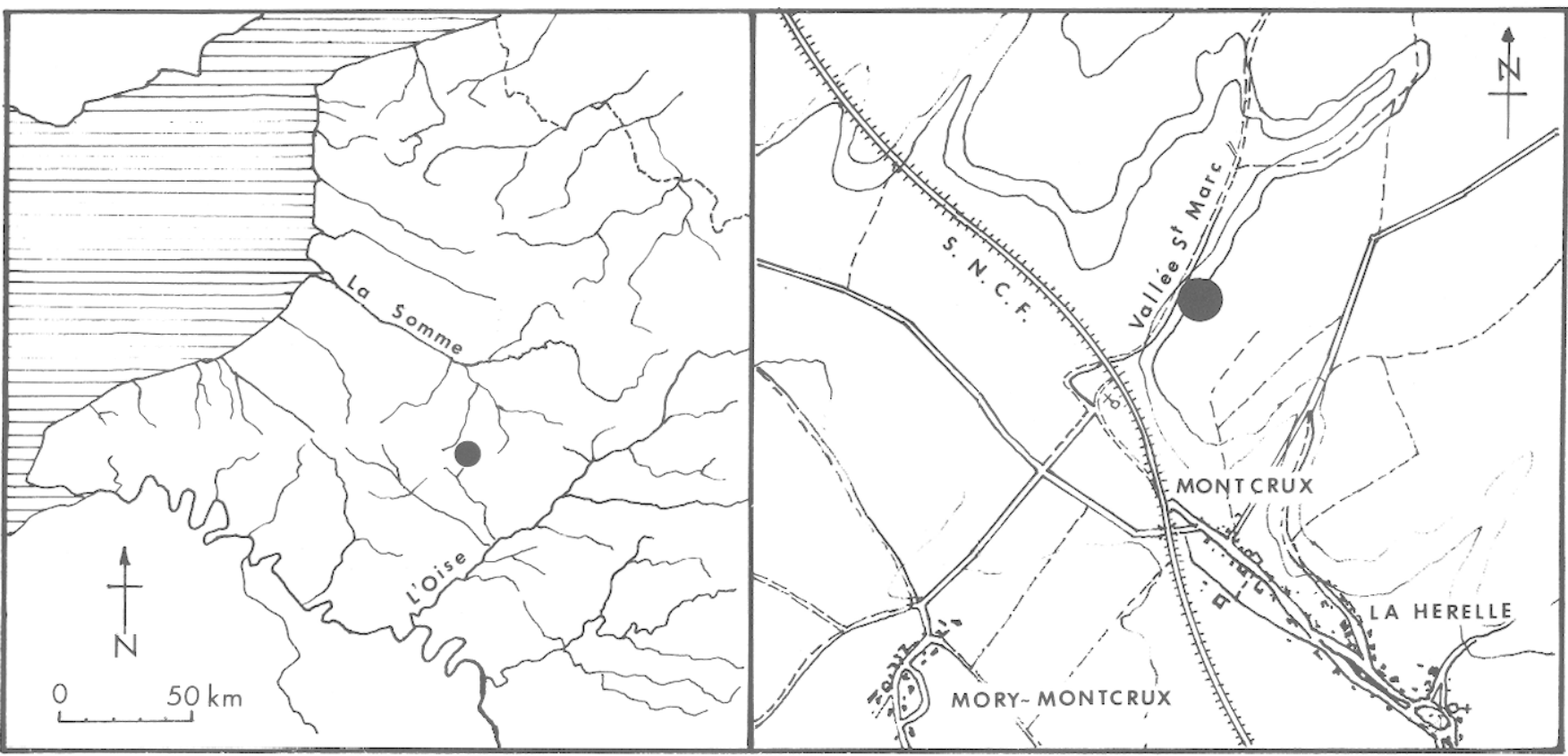

Fig. 1 - Situation de la nécropole gauloise de Mory-Montcrux (Oise) au lieudit «Les Vignes d'en Haut».

* Centre d'Etudes Techniques de l'Equipement Nord-Picardie et C.R.A.V.O. - 21 Rue des Cordeliers 60200 COMPIEGNE
La nécropole est implantée sur le bord de la vallée sèche de "Saint-Marc", près de l'importante ligne de chemin de fer Paris-Lille et du hameau de Montcrux. 
Sur le cadastre, la carrière est placée au lieudit "Sous les-Vignes-d'en-Haut (2) (fig. 2).

La petite nécropole occupe la mi-pente d'un mamelon de craie campanienne orientée sensiblement nord-est/ sud-ouest. Cette craie est assez tendre, fissurée et effritée en surface. On y remarque quelques lits de rognons de silex peu développés. La couverture superficielle est faible en raison de la pente (de $0,15 \mathrm{~m}$. à $0,20 \mathrm{~m}$. de lœess remanié). Dans ces conditions, le site est resté longtemps en friche, mais des essais d'agriculture (vignes du siècle dernier ?) ont perturbé la partie supérieure des sépultures. Actuellement, les lieux sont abandonnés par les cultivateurs. Une herbe rase et quelques buissons d'épineux tapissent les rebords de cette colline au relief peu marqué (fig. 3 ).

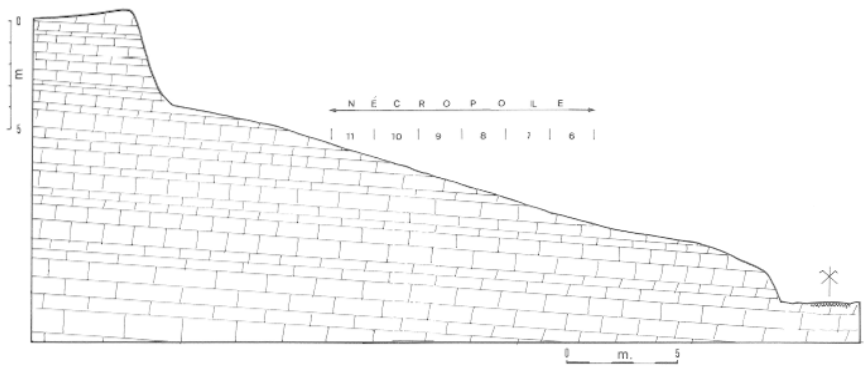

Fig. 3 - La nécropole gauloise de Mory-Montcrux. Profil en travers type permettant d'apprécier l'important pendage du terrain à l'emplacement des sépultures.

A près d'un kilomètre au nord de la nécropole de Mory-Montcrux, il a été découvert par prospection de surface un site d'habitat gaulois. Comme aucune fouille n'a été menée sur cet emplacement, nous ne pouvons pas savoir si des rapports existent avec la nécropole.

\section{LE PLAN DE LA NECROPOLE (fig. 4)}

La première sépulture mise au jour par l'agriculteur se trouve entre les carrés E 8 et F 8 . Les trois autres sépultures à incinération sont placées près du rebord de la carrière ; elles sont espacées à peu près tous les deux mètres. Les deux dernières sépultures sont des inhumations d'enfants qui se répartissent au même niveau que l'incinération 3 et sont situées respectivement à 1 mètre et 4,50 mètres de distance de celle-ci.

Les quatre tombes à incinération sont groupées et les deux sépultures à inhumation sont assez proches. Même si quelques sépultures ont été détruites par la carrière, nous pensons que la nécropole était peu importante (3).

\section{DESCRIPTION DES SEPULTURES}

\section{- La sépulture 0}

Elle a été entièrement détruite avant notre arrivée par les travaux de carrière. Des restes d'incinérations, les débris d'une coupe non reconstituable et un vase complet en proviennent. Nous avons nous-mêmes retrouvé quelques-uns de ces fragments sur le bord de la carrière (entre carrés E 8 et F 8).

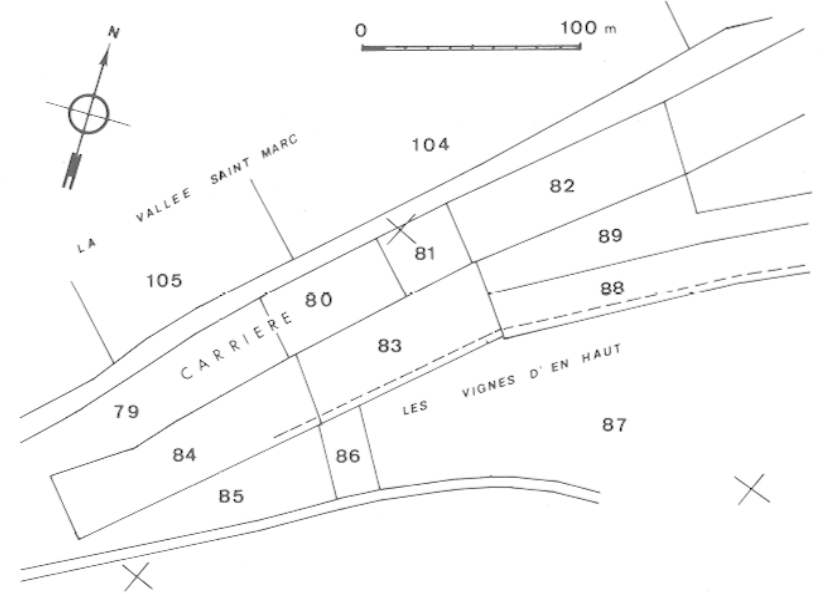

Fig. 2 - Plan cadastral de la nécropole gauloise de Mory-Montcrux (Oise) Section ZD, renouvelée en 1972.

- La sépulture 1 (fig. 5)

II s'agit d'une incinération trouvée dans le carré E 8, quadrant nord-ouest, à $0,25 \mathrm{~m}$ de profondeur. La tâche cendreuse a une forme ovalaire de $0,80 \mathrm{~m}$ sur $0,70 \mathrm{~m}$, de faible épaisseur. Quelques esquilles d'os très peu importantes proviennent du nord du milieu cendreux. Ces rares os semblent appartenir à un adulte. Les débris d'une fibule en fer et des morceaux de vases sont situés à la limite de la tâche cendreuse. Quelques petits tessons se trouvent à $0,50 \mathrm{~m}$ au nord-est des cendres.

- La sépulture 2 (fig. 6)

Elle est placée dans le carré F 9. C'est une inhumation d'un jeune enfant de 6-7 ans, orientée sensiblement est-ouest, la tête placée du côté du soleil couchant, dans le sens de la pente. Le crâne est fragmenté et seuls les gros os sont conservés. Le jeune individu se situe entre $0,30 \mathrm{~m}$ et $0,40 \mathrm{~m}$ de profondeur. On observe une légère distorsion du squelette au niveau du bassin.

Fig. 4 - Plan de la fouille de la petite nécropole de Mory-Montcrux (Oise) au lieudit "Les Vignes d'en Haut».

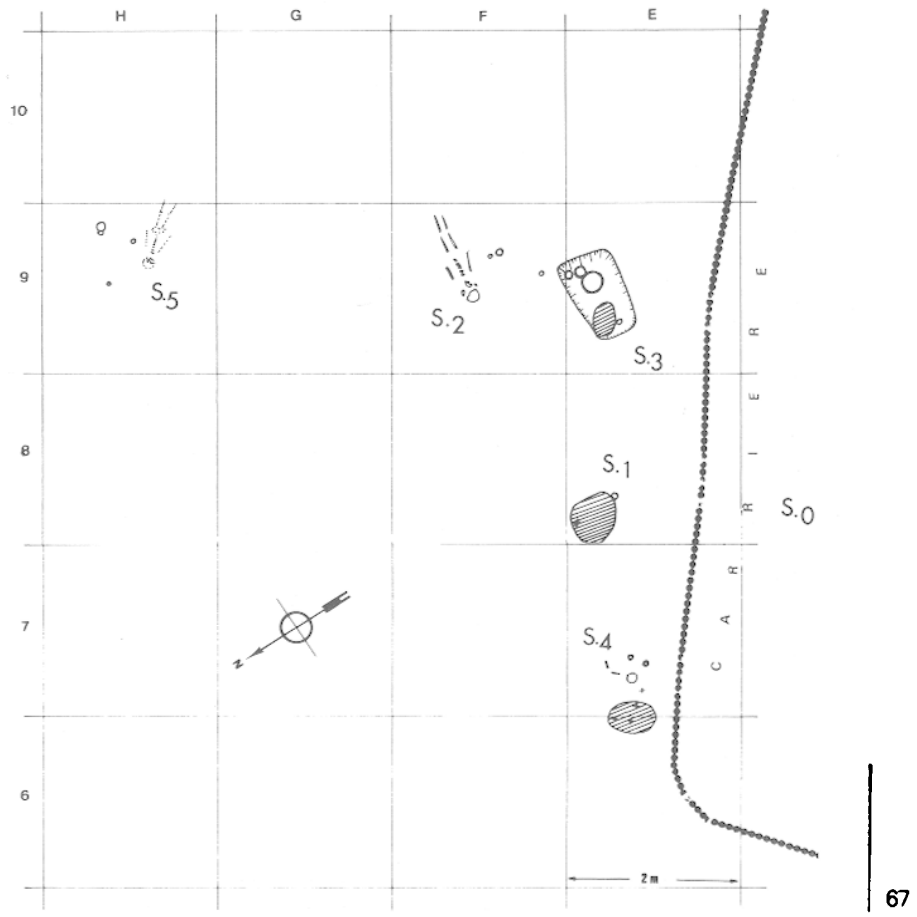




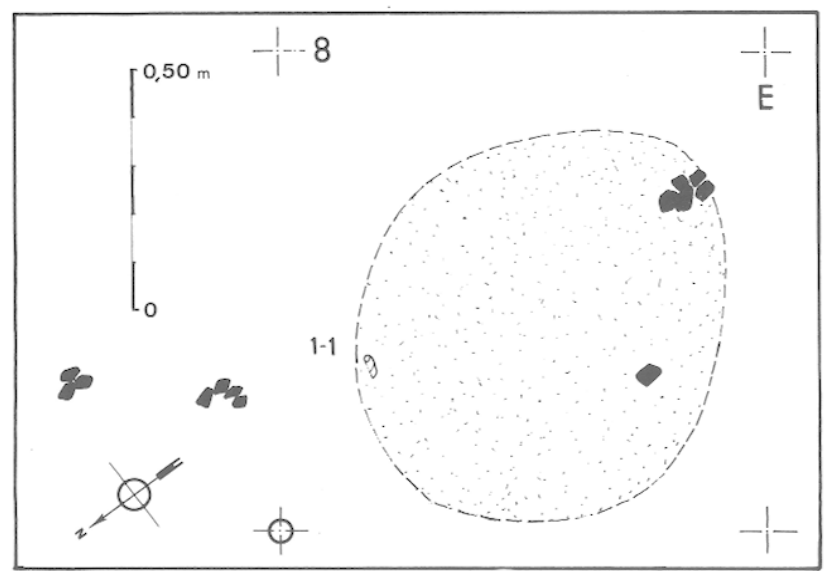

Fig. 5 - Mory-Montcrux (Oise), «Les Vignes d'en Haut». Plan de la sépulture à incinération $n^{\circ} 1$ - En pointillé, les vestiges de l'incinération et en noir, les tessons.

Des débris de vases sont répartis dans les labours autour du squelette. La plupart de ces tessons appartiennent à trois vases dont les parties inférieures ont été trouvées en place au sud du squelette

\section{- La sépulture 3 (fig. 7, 8 et 9)}

C'est la seule sépulture à incinération qui a fait l'objet d'un aménagement particulier. Une petite fosse presque rectangulaire, de $1,10 \mathrm{~m}$ sur $0,60 \mathrm{~m}$, creusée pour obtenir une assise horizontale, permet de mieux recueillir les vases à offrande. La paroi la plus haute mesure $0,30 \mathrm{~m}$ et la partie basse de la fosse rejoint la couche géologique de craie. L'incinération occupe le quart de la partie nord-ouest de la fosse. Une fibule, très rubéfiée, et quelques débris de gros os appartenant à un adulte sont inclus dans le centre de cette incinération formant une tâche ovalaire de $0,35 \mathrm{~m}$ sur $0,25 \mathrm{~m}$ (fig. 9). Un petit vase est placé sur le contour de la tâche cendreuse, presque dans l'axe de la fosse.

Fig. 7 - Mory-Montcrux (Oise) «Les Vignes d'en Haut» - Plan de la sépulture à incinération $n^{\circ} 3$.

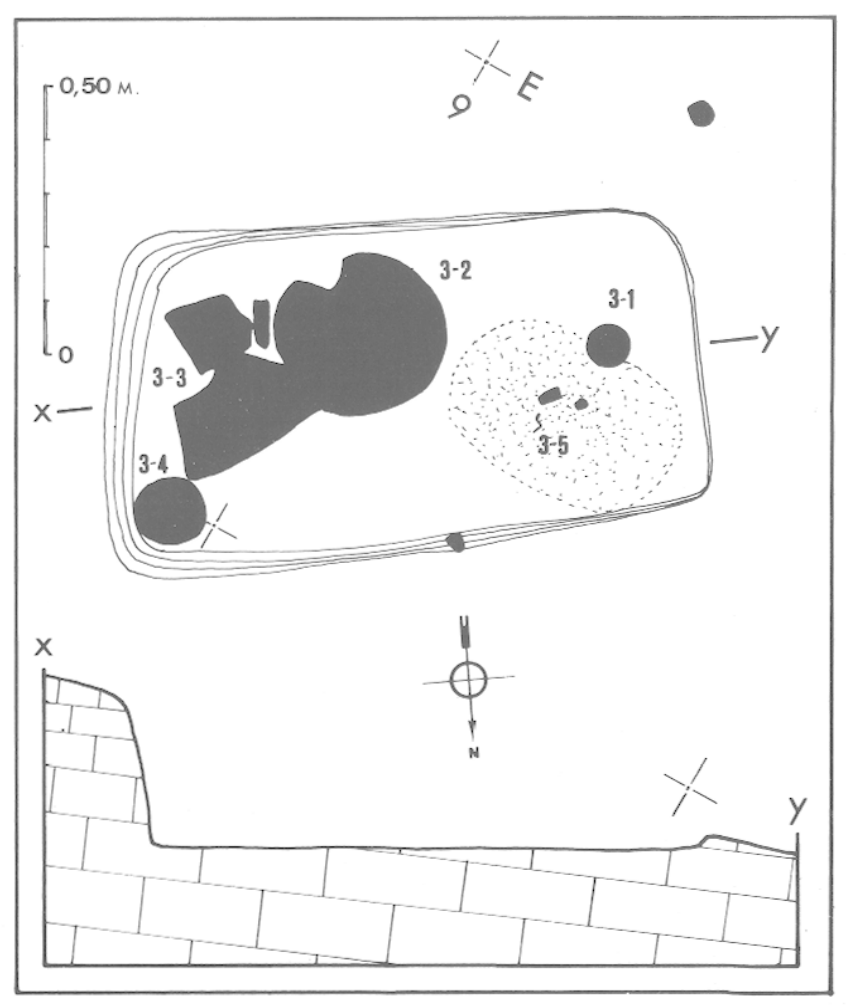

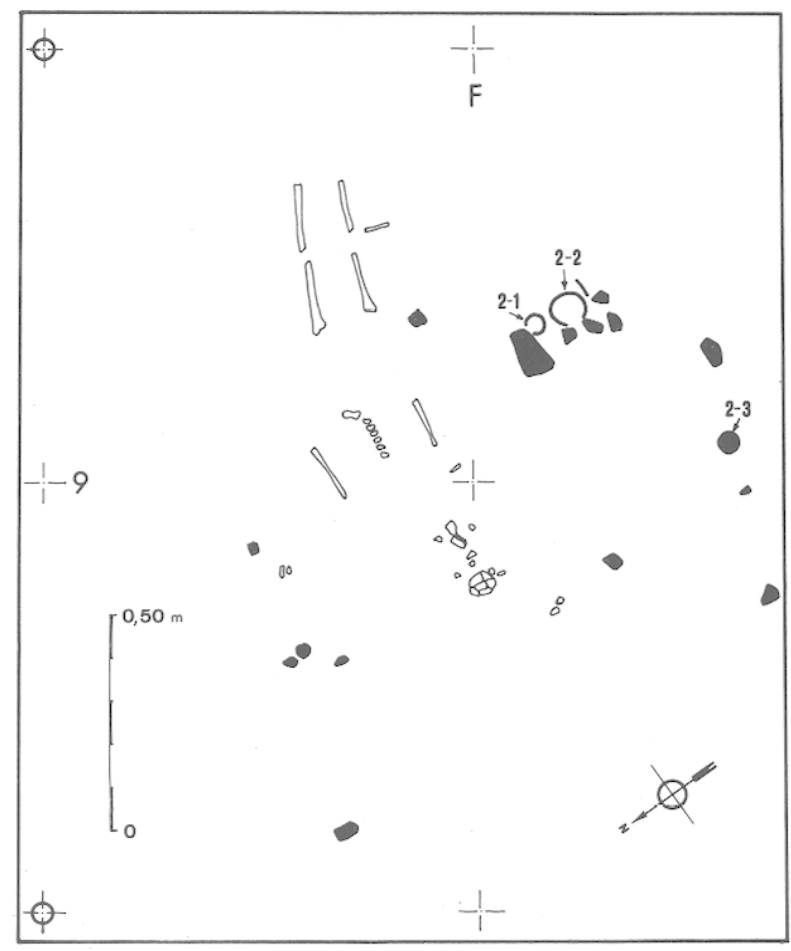

Fig. 6 - Mory-Montcrux (Oise) «Les Vignes d'en Haut» - Plan de la sepulture à inhumation $n^{\circ} 2$, d'un enfant.

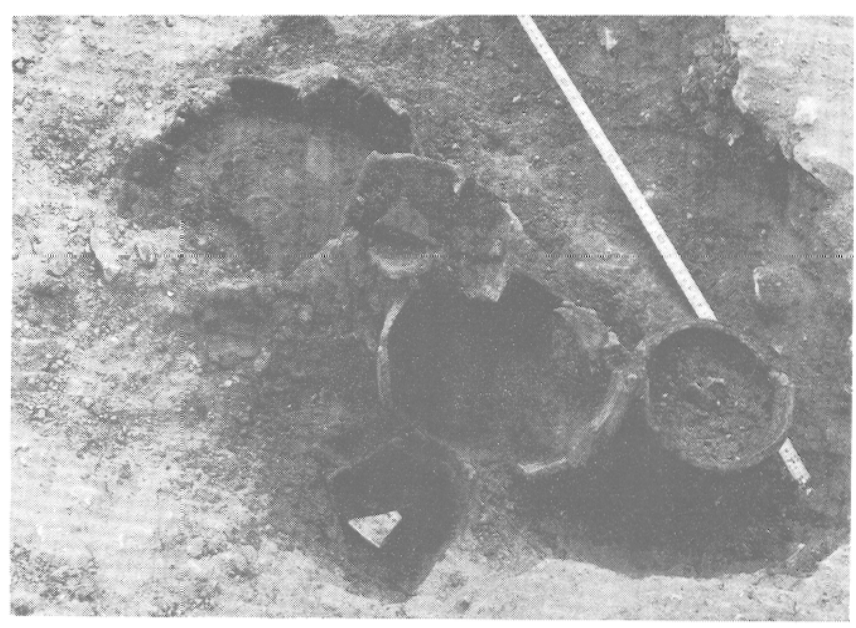

Fig. 8 - Mory-Montcrux (Oise) kLes Vignes d'en Haut" - Photographie des vases à offrande déposés dans la sépulture à incinération $n^{\circ} 3$.

Fig. 9 - Mory-Montcrux (Oise) "Les Vignes d'en Haut». Détail montrant le mauvais état de conservation des restes osseux incinérés, provenant de la sépulture $n^{\circ} 3$.

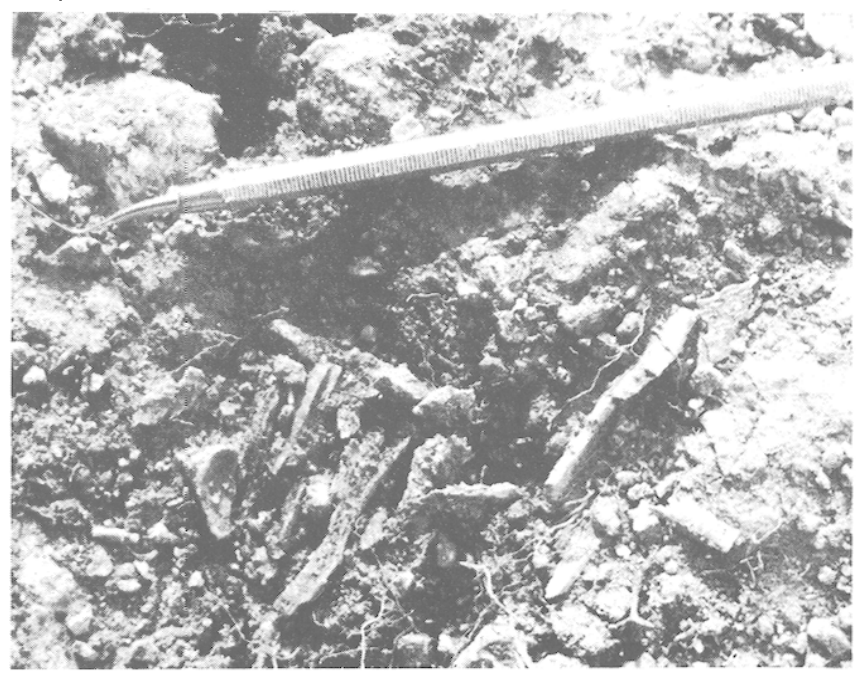


Deux vases hauts et une coupe assez bien conservés se trouvent dans la deuxième moitié supérieure de la fosse (fig. 8).

- La sépulture 4 (fig. 10)

Cette incinération est à la jonction des carrés E7 et R6. Elle est très détériorée par les anciens travaux agricoles, vue sa faible profondeur $(0,25 \mathrm{~m})$. La tache cendreuse de l'incinération est ovalaire, de $0,75 \mathrm{~m}$ sur $0,60 \mathrm{~m}$ environ. Un petit amas d'os calcinés d'humain adulte se trouve dans la partie superieure, ainsi que trois fibules en fer. Trois vases, très détériorés, et un anneau de bronze sont placés entre $0,50 \mathrm{~m}$ et $1,10 \mathrm{~m}$ au sud-est de l'incinération. L'état de fragmentation de ces vases n'a pas permis de reconstitution ultérieure.

- La sépulture 5 (fig. 11)

Elle est placée à $0,30 \mathrm{~m}$ de profondeur dans le carré H 9. II s'agit indiscutablement d'une inhumation de jeune enfant (3-4 ans) qui a été bouleversée par les labours.

Le squelette n'est plus représenté que par des débris de la boîte crânienne et de la mandibule inférieure, et par quelques gros os très fragmentés. Les fragments de trois vases sont placés à $1 \mathrm{~m}$ au nord-est du squelette.

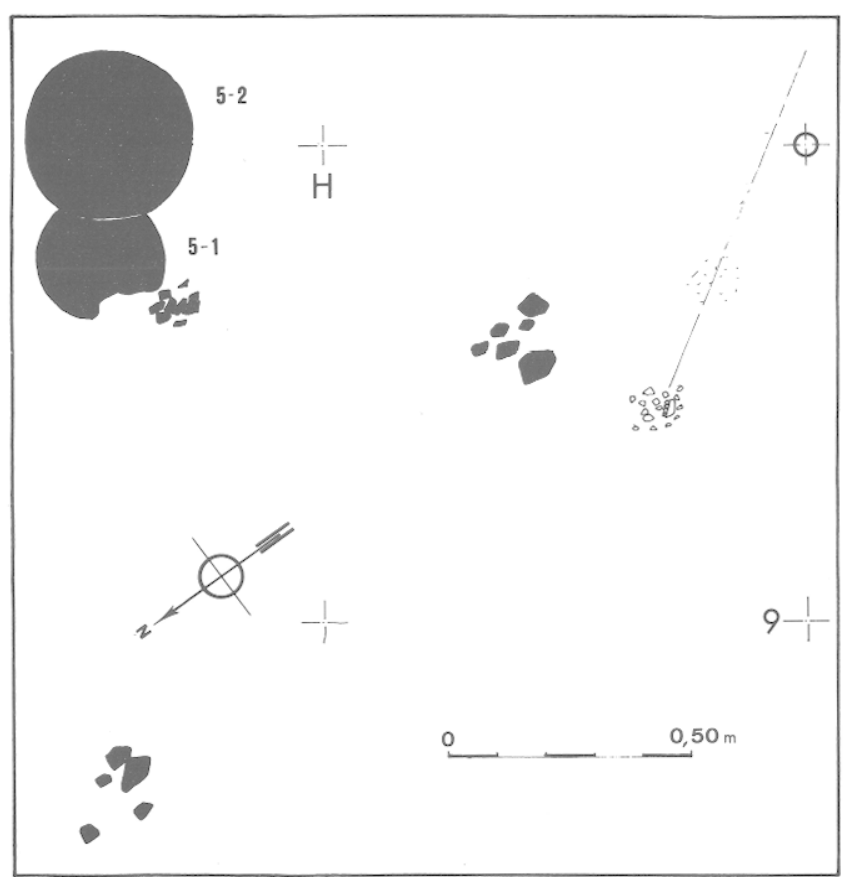

Fig. 11 - Mory-Montcrux (Oise), "Les Vignes d'en Haut» - Plan de la sépulture à inhumation $n^{\circ} 5$, d'un jeune enfant.

\section{LE MATERIEL ARCHEOLOGIQUE}

- La sépulture 0. Incinération ?

0 - 1 - Petit pot tronconique mis au jour par le cultivateur (fig. $12, n^{\circ} 0$ ). II a été brisé au niveau du col par la pelle mécanique. D'excellente cuisson, il a une couleur extérieure brun grisâtre. La lèvre est arrondie et légèrement sortante. Un sillon bien marqué est appliqué au-dessus de la panse.

$\mathrm{Au}$ milieu de la panse, on distingue une bande horizontale de $48 \mathrm{~mm}$ de largeur, exécutée par râclage de la pâte afin de lui donner un aspect mat qui contraste avec le lissage brillant du reste du vase.

(H. t. : $138 \mathrm{~mm}$; d. fond : $75 \mathrm{~mm}$; d. panse : $118 \mathrm{~mm}$; d. ouverture : $104 ; \mathrm{H}$. du fond de la panse : $97 \mathrm{~mm}$ ). 0 - 2 - Débris non reconstituables d'une coupe a fond

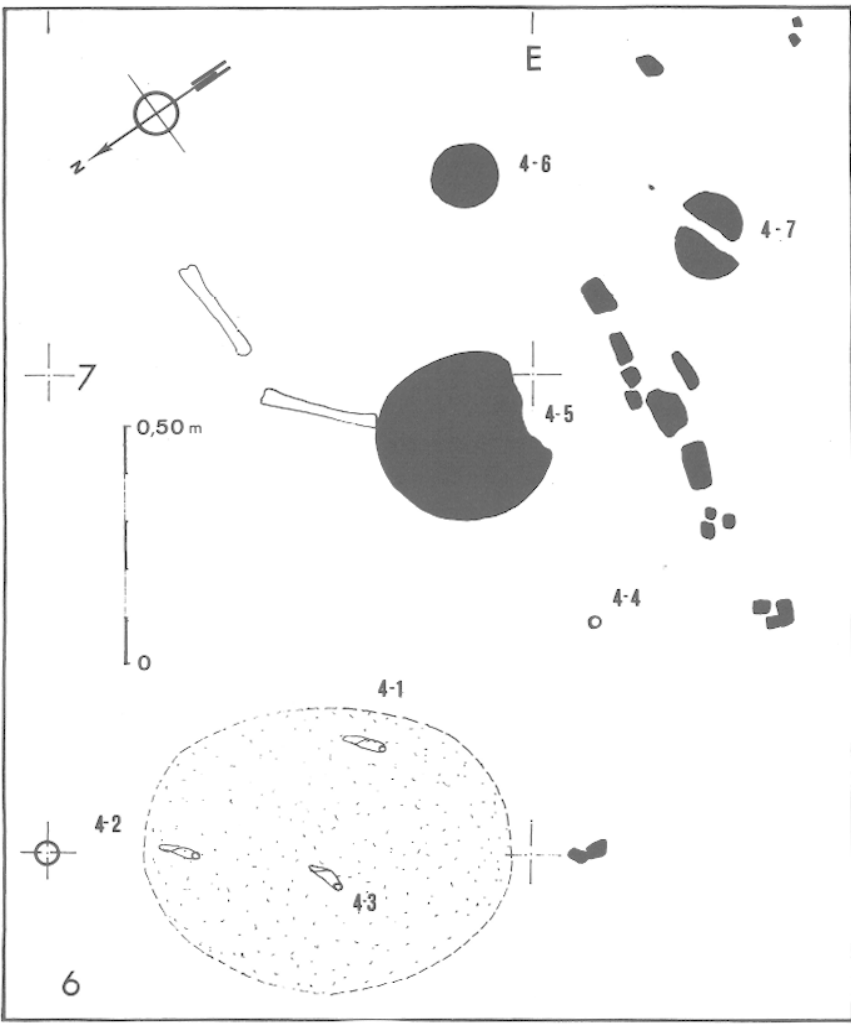

Fig. 10 - Mory-Montcrux (Oise) "Les Vignes d'en Haut" - Plan de la sépulture à incinération $n^{\circ} 4$.

arrondi en terre noirâtre qui a été trouvée dans les déblais de la carrière.

- La sépulture 1. Incinération

1 - 2 et 1 - 3 - Seuls quelques fragments de vase ont été trouvés dans cette sépulture très endommagée par les anciens labours, ou tout près d'elle. Deux ensembles de tessons semblent correspondre à des coupes à fond arrondi en terre noirâtre.

1 - 1 - Les débris d'une fibule en fer rubéfiée proviennent de l'incinération (fig. 14, $n^{\circ} 1-1$ ). Le ressort a disparu et il ne subsiste que l'ardillon et l'arc. Le porte-ardillon est formé par le retour du fil martelé sur l'arc, maintenu par aplatissement de l'extrémité qui se rabat autour de l'arc.

- La sépulture 2. Inhumation d'enfant Trois vases appartiennent à cette sepulture : 2 - 1 - Petit pot bitronconique, caréné, à pâte de couleur extérieure brun-rougeâtre sur le dessus et noirâtre en-dessous. Le dégraissant est peu visible et le lissage bien soigné. Ce vase est légèrement dissymétrique (fig. 12, $\mathrm{n}^{\circ}$ 2-1) (H. t. : $53 \mathrm{~mm}$; d. fond : $38 \mathrm{~mm}$; d. panse : $68 \mathrm{~mm}$; d. ouverture : $52 \mathrm{~mm} ; \mathrm{H}$. du fond à la carène : $38 \mathrm{~mm}$ ).

2 - 2 - Vase tronconique à col rentrant, iégèrement évasé. La lèvre est arrondie. La pâte a une couleur extérieure brunâtre-noirâtre. La surface est bien lissée et offre un aspect brillant. La moitié de la panse est décorée horizontalement de neuf rangées, d'incisions exécutées à cru, dans un style assez désordonné (fig. 12, $n^{\circ}$ 2-2). Ces impressions triangulaires, très allongées, semblent avoir été exécutées avec l'extrémité d'un couteau. (H. t. : $154 \mathrm{~mm}$; d. prof. : $88 \mathrm{~mm}$; d. ouverture : $142 \mathrm{~mm}$; d. panse : $148 \mathrm{~mm} ; H$. fond à la carène : $111 \mathrm{~mm}$ ).

2 - 3 - Fragment inférieur d'un vase à piédestal. II a une couleur extérieure noirâtre et une surface bien 

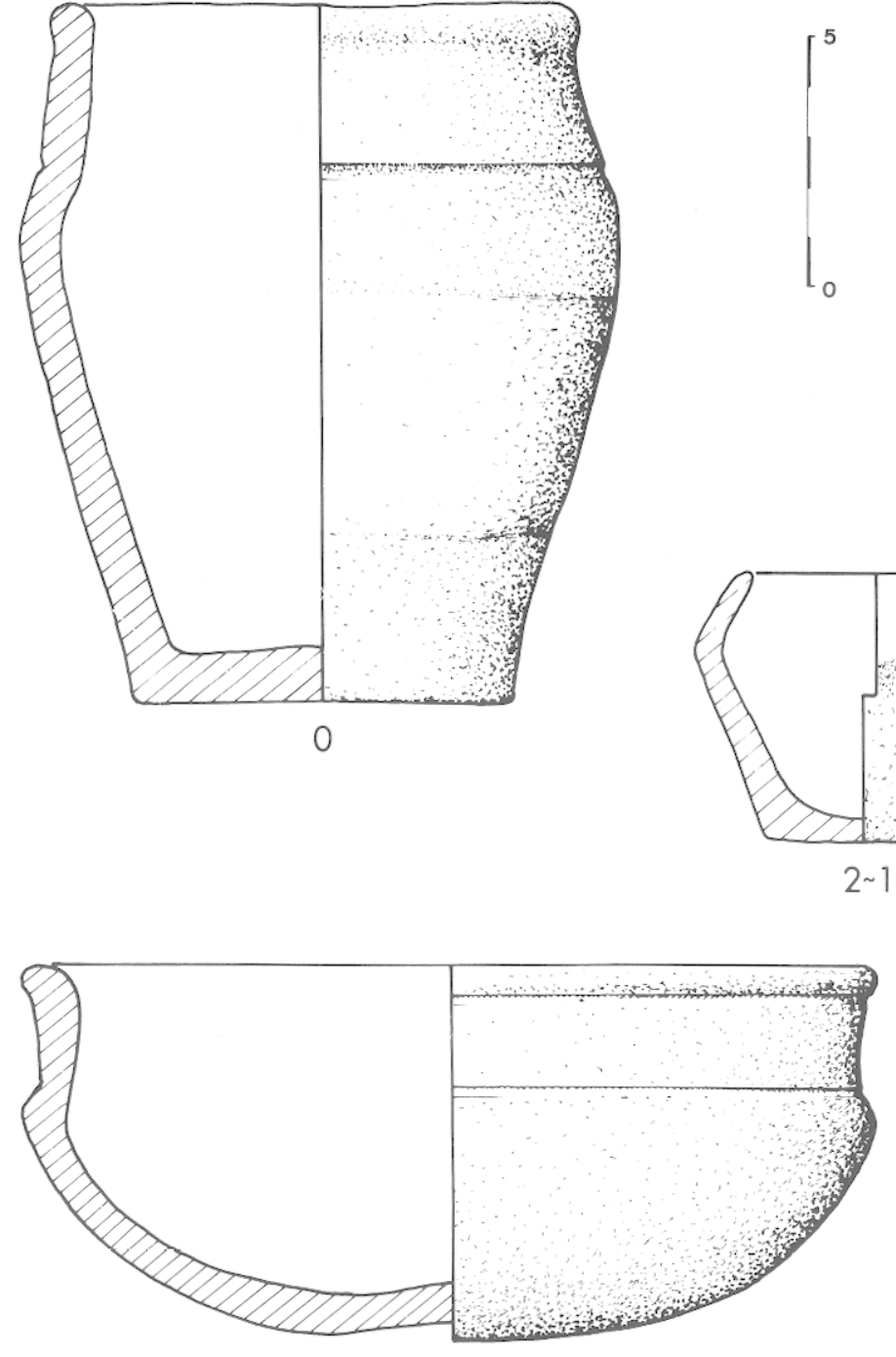

$5 \sim 1$

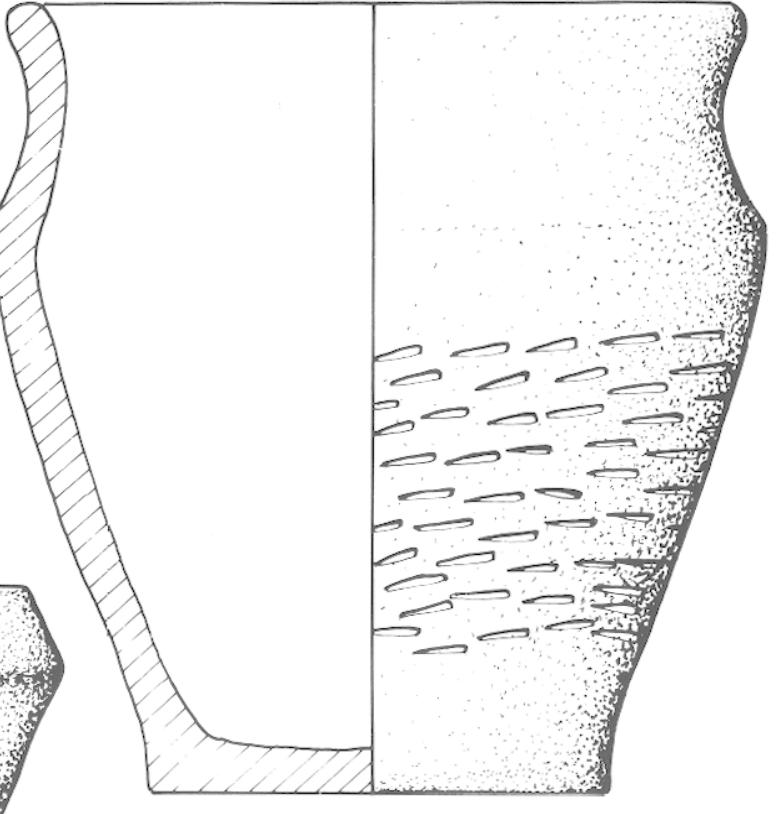

2 2

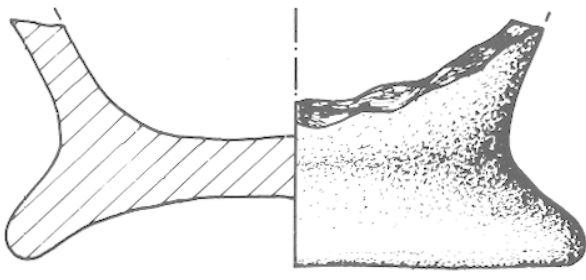

2 3

Fig. 12 - Mory-Montcrux (Oise) - «Les Vignes d’en Haut» - Le matériel céramique trouvé dans les sépultures 0, 2 et 5.

lissée et brillante (fig. 12, $\mathrm{n}^{\circ}$ 2-3). (d. du pied : $112 \mathrm{~mm}$; d. du fond : $87 \mathrm{~mm}$ ).

\section{- La sépulture 3. Incinération}

L'encaissement de cette sépulture a bien protégé les vases.

3 - 1 - Petit godet en forme de tonnelet. II a une pâte de couleur extérieure brun orangée, avec, dans la partie basse des plages ocrées. II est soigneusement lissé, mais non brillant (fig. $13, n^{\circ} 3-1$ ).

(H. t. : $80 \mathrm{~mm}$; d. fond : $48 \mathrm{~mm}$; d. panse : $88 \mathrm{~mm}$; d. ouverture : $68 \mathrm{~mm}$ ).

3 - 2 - Grande coupe à fond ombiliqué. Le col est légèrement sortant. La lèvre arrondie est légèrement épaissie. La pâte est bien lissée et a un aspect brillant. La couleur extérieure est brun noirâtre. Elle est décorée de deux sillons horizontaux placés en haut de la panse et sous le rebord de la lèvre (fig. 13, $n^{\circ} 3-2$ ).

(H. t. : $129 \mathrm{~mm}$; d. ouverture : $360 \mathrm{~mm}$; d. panse : $257 \mathrm{~mm} ; \mathrm{H}$. du fond de la panse : $73 \mathrm{~mm}$ ).

3 - 3 - Grand vase à panse ovoīde et à col légèrement rentrant dont la surface extérieure est brun noir. Le milieu de la bande horizontale de $96 \mathrm{~mm}$ de largeur, obtenue par grattage de la pâte sèche à l'aide d'un peigne à 6 dents (fig. 13, $n^{\circ} 3-3$ ). Ce grand vase est un peu dissymétrique. (H. t. : $278 \mathrm{~mm}$; d. fond : $111 \mathrm{~mm}$; d. ouverture : $192 \mathrm{~mm}$; d. panse : $223 \mathrm{~mm}$; H. fond à la panse : $187 \mathrm{~mm}$ ).

3-4 - Vase tronconique à col légèrement rentrant et à lèvre soulignée par un épaississement peu marqué. Le fond est un peu concave dans sa partie centrale. II a un état de surface bien lissé, brillant et une pâte extérieure de couleur noirâtre.

II n'est pas décoré (fig. 13, n० 3-4).

(H. t. : $184 \mathrm{~mm}$; d. fond : $92 \mathrm{~mm}$; d. ouverture : $149 \mathrm{~mm}$; d. panse : $156 \mathrm{~mm} ; \mathrm{H}$. fond à la panse : $142 \mathrm{~mm}$ ).

3-5 - Fragment d'arc de fibule en fer, probablement du même type que les autres (fig. 14, $n^{\circ} 3-5$ ).

- La sépulture 4. Incinération

4-5 - Grande coupe à fond ombiliqué, très fragmenté par les labours. Elle n'a pu être reconstituée. Le diamètre de la panse mesure environ $340 \mathrm{~m}$. Elle semble avoir une forme proche de celle trouvée dans la sépulture $3, \mathrm{n}^{\circ} 2$.

4-6 - Fond de vase tronconique en céramique de couleur brun-noirâtre (fig. 10) $(150 \mathrm{~mm})$.

4-7 - Fond de vase tronconique, proche du précédent (diamètre du fond : $120 \mathrm{~mm}$ ). 
4-4 - Petit anneau en potin de $22 \mathrm{~mm}$ de diamètre, à section sub-losangique. Une usure se dessine sur un côté.

4-3 - Fragment de fibule en fer, à ressort à 5 spires et corde extérieure (fig. 14, $\mathrm{n}^{\circ} 4-3$ ).

4-2 - Fragments d'un ardillon et d'un arc de fibule en fer. Le porte ardillon se referme au sommet de l'arc par un aplatissement et un enroulement (fig. 14, $n^{\circ} 4-2$ ). 4-1 - Fibule en fer à corde extérieure et à arc constitué de cinq spires. Le porte ardillon se referme aussi sur l'arc (fig. 14, $\mathrm{n}^{\circ}$ 4-1). Cette fibule, bien qu'ayant aussi subi la crémation, est la seule mesurable (Longueur : $76 \mathrm{~mm}$; largeur corde : $20 \mathrm{~mm}$; $\mathrm{H}$. : $19 \mathrm{~mm}$ ).

Fig. 13 - Mory-Montcrux (Oise), «Les Vignes d’en Haut» - Le matériel céramique provenant de la sépulture à incinération n 3 . En 3-5, fragment de fibule.

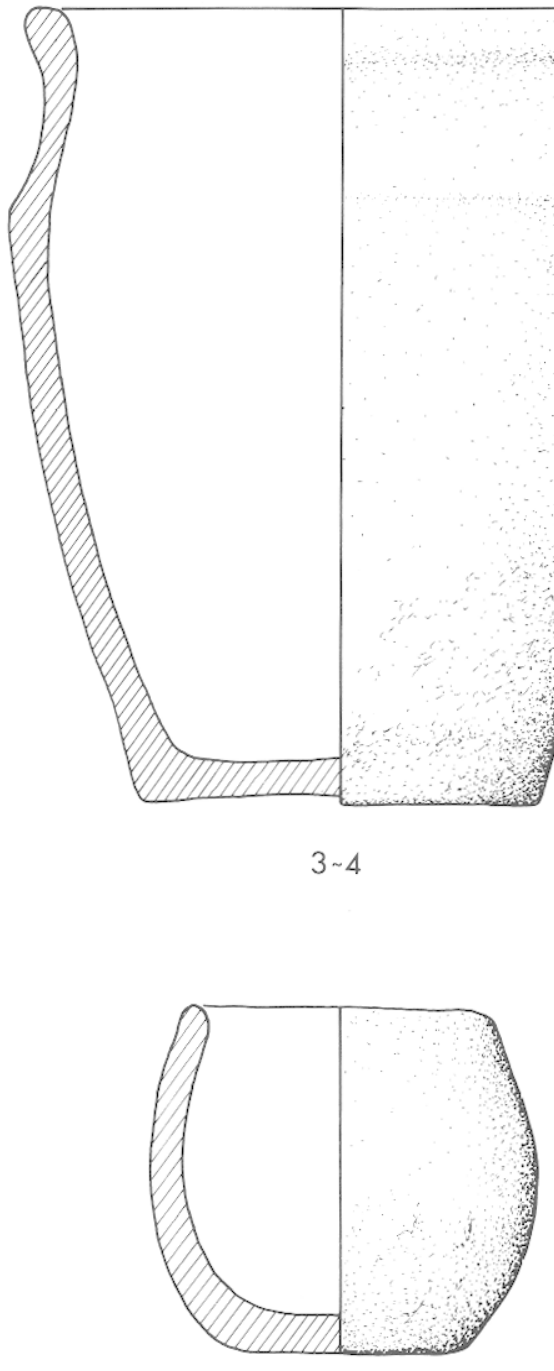

3 1

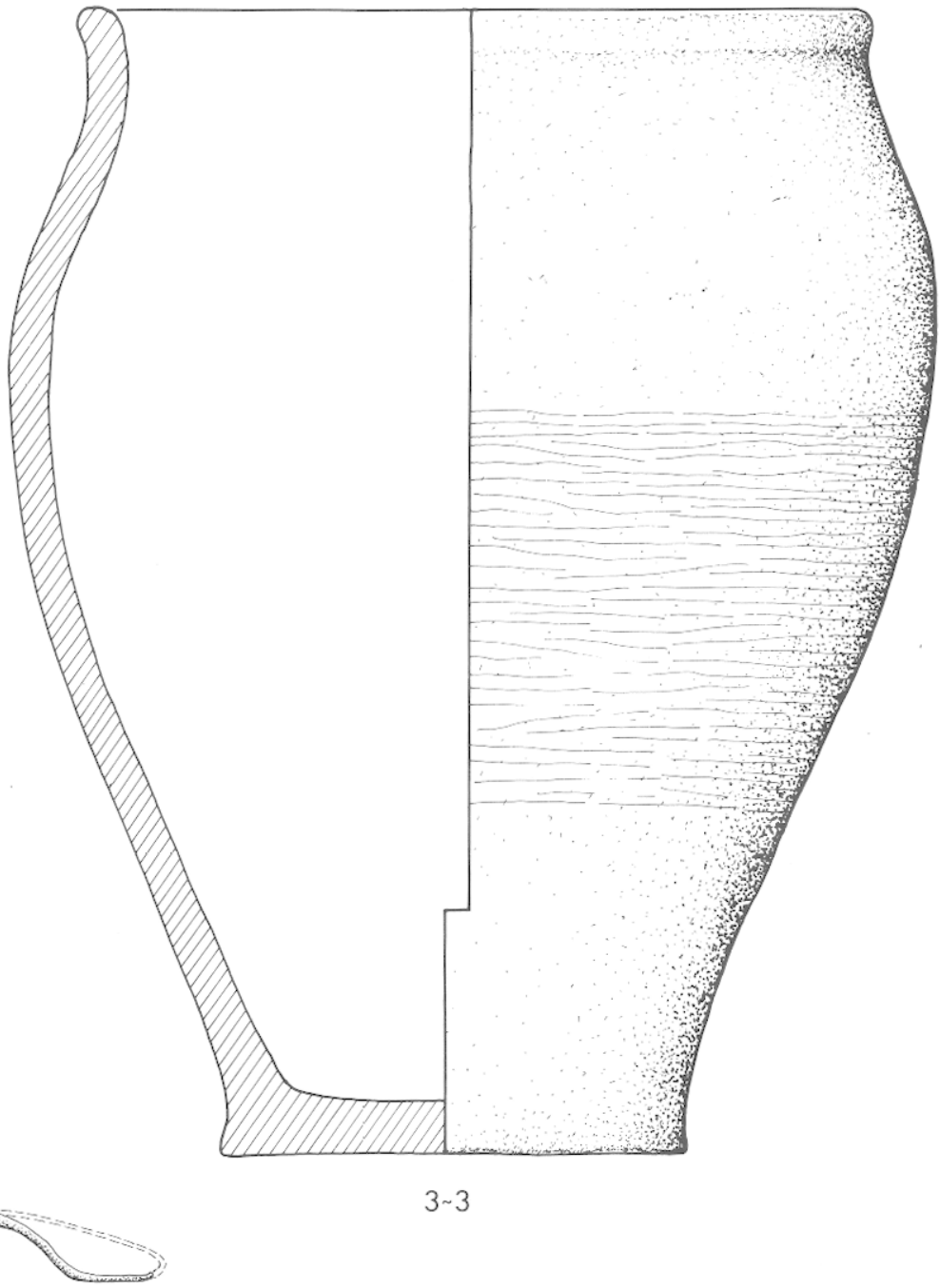

$3 \sim 5$

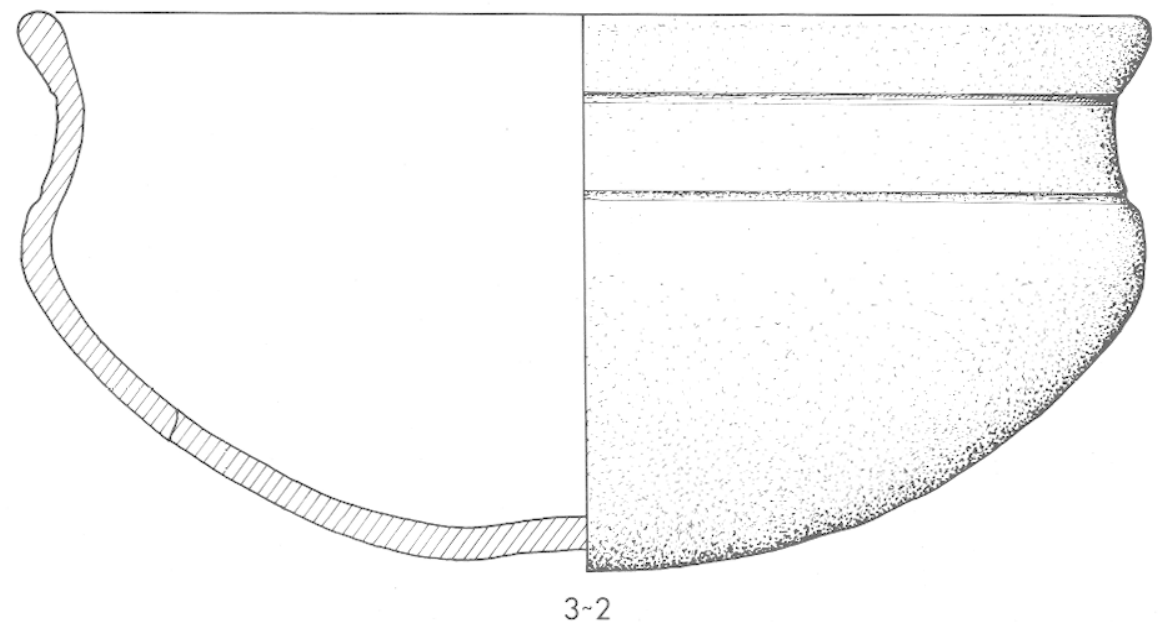



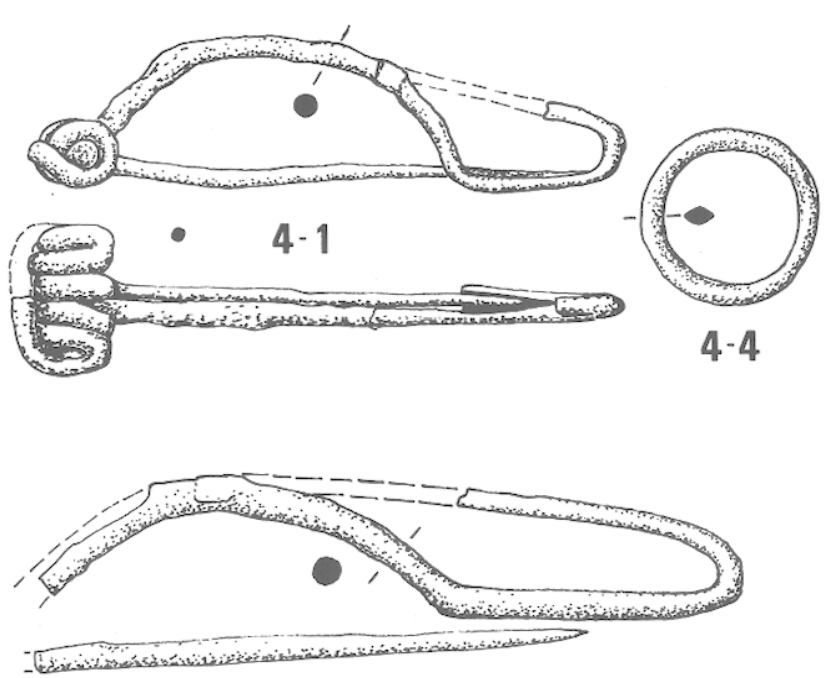

4 - 2
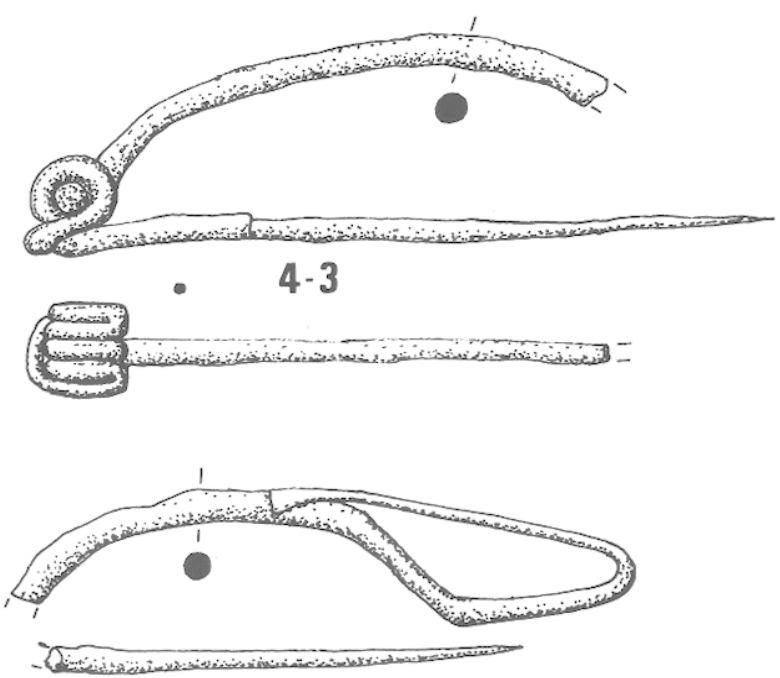

$1-1$
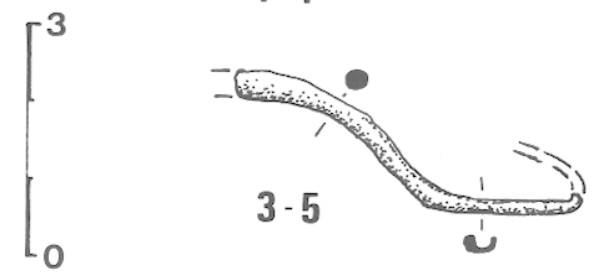

Fig. 14 - Mory-Montcrux (Oise), «Les Vignes d'en Haut» 1 à 5, fibules en fer ; 6 , anneau en bronze $\left(1\right.$ a 3 , sépulture $n^{\circ} 4 ; 4$, sépulture $n^{\circ} 1 ; 5$, sépulture $n^{\circ} 3$ et 6 , sépulture $n^{\circ} 41$.

- La sépulture 5 - Inhumation d'enfant 5-1 - Coupe à fond ombiliqué et à col légèrement rentrant, souligné par un épaississement de la lèvre. Elle est décorée de deux rainures horizontales placées sous la lèvre et en haut de la panse (fig. 12, n 5-1). Elle est soigneusement lissée et a une couleur extérieure brun noirâtre.

(H. t. : $75 \mathrm{~mm}$; d. ouverture : $168 \mathrm{~mm}$; d. panse : $168 \mathrm{~mm} ; \mathrm{H}$. du fond à la panse : $44 \mathrm{~mm}$ ).

5-2 - Coupe entièrement émiettée, non reconstituable. Le diamètre de la panse est de $240 \mathrm{~mm}$ environ. Elle a une pâte de couleur noirâtre.

\section{COMPARAISONS ET CONCLUSION.}

Les six sépultures de Mory-Montcrux ont livré un mobilier homogène qui les place dans une tranche d'âge assez proche. Le rite de l'incinération est réservé, semble-t-il, aux adultes, alors que les deux enfants sont inhumés. II existe donc simultanément l'utilisation des deux rites. Dans la nécropole voisine de Breuil-leSec (Oise), l'incinération est de règle (DEGENNE M., 1978 et DUVAL A., rapports de fouilles). Dans la nécropole de La Tène II finale d'Allonville (Somme), les deux sépultures complètes ont aussi livré des incinérations avec offrandes (FERDIERE A. et alii, 1973). A Port-le-Grand (Somme), les rares indications laissées par le fouilleur attestent aussi le rite de l'incinération (LEMAN DELERIVE G., 1976).

Dans l'Oise, les vieilles fouilles d'Emmanuel WOILLEZ sur le site du "Tremblaye» à Verneuil-en-Halatte, ont permis de mettre au jour une dizaine de sépultures à inhumation. Le matériel recueilli est datable de la fin de La Tène ancienne (DURVIN P., 1963). L'incinération n'est pas du tout mentionnée dans cette nécropole.

Au «Hameau de Saint-Maur» à Gournay-sur-Aronde (Oise), Albert de ROUCY a fouillé en 1869, deux sépultures à inhumation et deux incinérations. Le matériel recueilli est proche de celui de Breuil-le-Sec et attribuable à La Tène II (BLANCHET J.-Cl. et DUVAL A., 1975). Toujours à Gournay-sur-Aronde, mais cette fois-ci, dans l'oppidum et le sanctuaire gaulois du "Parc du Château», Jean-Louis BRUNAUX a fouillé un dépôt de vases entreposés dans une fosse quadrangulaire qui fut interprétée au début comme une sépulture de La Tène Ic.

En raison de l'absence d'incinération et des résultats de la fouille du sanctuaire qui s'en est suivie, ce dépôt semble plutôt rituel (BRUNAUX J.-L., 1975).

Du matériel proche de celui de Mory-Montcrux provient d'une sépulture à incinération trouvée au «Hameau de Beaulieu», à Baron (Oise) (BLANCHET J.-Cl. et DUVAL A., 1975, p. 55 ; fig. $6, n^{\circ}$ 5). A La Tène finale le rite de l'incinération est de règle comme nous en avons quelques échos à travers les vieilles fouilles menées en forêt de Compiègne au "Puits Féron» et aux "Secneaux» (BLANCHET J.-Cl. et DUVAL A., 1975).

Deux sépultures à incinération de La Tène tardive ont été exhumées sous les vestiges d'une installation gallo-romaine au "Houy», à Creil (Oise) (DURVIN P., 1968 et 1981).

La petite nécropole de Mory-Montcrux se situe dans la période où le rite de l'incinération dans la région peu à peu la règle générale à partir du début de La Tène II.

Les comparaisons céramiques nous écartent quelque peu des séries de vases livrées dans les nécropoles de La Tène II, de Breuil-le-Sec (Oise) (DEGENNE M., 1978), de Gournay-sur-Aronde, "Le Parc» (BLANCHET J.-Cl. et DUVAL A., 1975), d'Allonville (Somme) (FERDIERE et alü, 1973) et de Port-le-Grand (Somme) (LEMAN DELERIVE G., 1976).

Les vases bitronconiques de Mory-Montcrux ramènent en particulier vers des formes plus carénées, situliformes. Les vases en question (fig. $12, n^{\circ} 2-2$ et fig. 13 , 
$\mathrm{n}^{\circ}$ 3-4) trouvent des comparaisons régionales à Baron (Oise) (BLANCHET J.-Cl. et DUVAL A., 1975, fig. 6 , $n^{\circ} 5$ ) et à Sandrancourt (Val d'Oise) (SIMON Ph., 1969 et 1973). A Baron, le vase possède aussi un décor d'impressions triangulaires très allongées, faites peutêtre aussi avec la pointe d'un couteau. A Sandrancourt, Philippe SIMON donne le dessin d'un vase pratiquement identique aux deux vases de Mory-Montcrux. Le même auteur établit des comparaisons avec un vase proche provenant d'une sépulture à inhumation féminine de La Tène I b-c de la Marne à Caurel "La Fosse Minore». Dans l'abondante littérature concernant les nécropoles de la Marne, on retrouve quelques vases assez proches dans des ensembles datés de La Tène ancienne de phase récente.

Les fibules de Mory-Montcrux sont toutes de type simple en fer, dont le modèle a pu perdurer quelque temps. Elles ont une corde extérieure et un porte ardillon qui se referme sur le début du sommet de l'arc. Ce modèle de fibule se rencontre aussi dans la Marne à la fin de La Tène ancienne et surtout au début de La Tène II.

Toutes ces considérations permettent d'envisager pour Mory-Montcrux une place chronologique se situant à la fin de La Tène ancienne ou au tout début de La Tène II, avant les sépultures les plus anciennes de la nécropole de Breuil-le-Sec. La petite quantité de sépultures mises au jour à Mory-Montcrux, même en tenant compte de quelques destructions avant notre arrivée, fait penser à une petite communauté autarcique, peut-être familiale.

\section{NOTES}

(1) Nous tenons à remercier vivement Monsieur MAILLARD pour toutes les informations et autorisations qu'il put nous fournir, ainsi que la Direction des Antiquités Historiques, qui nous a accordé le sauvetage de cette nécropole. La fouille a été menée par une petite équipe constitúée de : Annie BARBASTE, Annie GERVAIS, Michel DEGENNE, Roger JACQUINEZ, Laurence et Jean-Louis BRUNAUX, Jean-Luc CABARES, Michel DUPONT et Christophe TOUPET.

(2) Le cadastre a été renouvelé en 1972. Le site est placé sur la parcelle $n^{\circ} 80$ de la Section ZD (anciennement Section A, $n^{\circ}$ 543-544). Les coordonnes Lambert du site sur la carte au 1/25 000 de Saint-Just-enChaussée, de I'I.G.N., sont $x: 604,9 ; y: 211,3$ et $z: 120$ a $125 \mathrm{~mm}$.

(3) M. MAILLARD n'a rien observé avant la découverte du premier vase intact. Il n'a pas le souvenir de fosse ou de zone cendreuse.
(4) Nous exprimons toute notre gratitude à l'Institut de Recherche Archéologique et Paléométallurgique de Compiègne qui s'est chargé de la restauration des quelques objets métalliques.

\section{BIBLIOGRAPHIE}

BLANCHET J.-Cl. et DUVAL A. (1975). - "Les collections de La Tène provenant de l'Oise et de la Somme au Musée des Antiquites Nationales». Antiquités Nationales, 7, p. 49-58, 7 fig.

BLANCHET J.-Cl. et DUVAL A. (1976). - "Le deuxième Age du Fer ou époque de La Tène, en Picardie». Revue Archóologique de l'Oise, 7, p. 48-58, 8 fig.

BLANCHET J.-Cl. et JACQUINEZ R. (1975). - «Fouilles de sauvetage de la nécropole gauloise de Mory-Montcrux (Oise). Note préliminaire»). Revue Archéologique de l'Oise, 5, p. 34-37, 11 fig.

BLANCHET J.-Cl, BUCHSENSCHUTZ O et MENIEL P. (1983) - "La maison de La Tène moyenne de Verberie (Oise). Actes du 5 ème colloque de Senlis sur le Second Age du fer dans l'Oise et le Nord de la région parisienne». Revue Archóologique de Picardie, numéro spécial, 1.

BIRCHALL A. (1965) - "The Aglesford - Swarling Culture : the problem of the Belgae reconsidered» - Procedings Prehistoric Society, 31, p. 241-367.

BRUNAUX J.-L. (1975) - «Sépulture de La Tène II à Gournay-surAronde (Oise)"). Revue Archélogique de /'Oise, 6, p. 27-31; 9 fig.

BRUNAUX J.-L., MENIEL P. et RAPIN A. (1980) - "Un sanctuaire gaulois à Gournay-sur-Aronde (Oise)» - Gallia, 38, fasc. 1, p. 1-25, 25 fig. DEGENNE M. (1978) - "La nécropole gauloise, de Breuil-le-Sec, sondage réalisé en 1974». Mémoires de la Société Archéologique et Historique de Clormont-on-Beauvaisis, t. XXXIV, p. 1-14, 4 fig.

DURVIN P. (1963) - «Sépultures du Second Age du fer à Verneuil-enHalatte (Oise)». Cefticum, VI, p. 103-111.

DUVAL A. (1976). «Aspects de La Tene moyenne dans le Bessin Parisien». Bull. soc. Préhist. Française, 73, Etudes et Travaux, p. 457-484, 21 fig.

DUVAL A. et BUSCHENSCHUTZ O. (1976). - "L'Age du fer dans le Bassin Parisien et le Nord de la Francen. La Préhistoire Française, t. II, p. 789-801.

FERDIERE A. et ahü (1973). - «Les sépultures gauloises d'Allonville (Somme)». Bull. Soc. Préhist. Française, 70, Etudes et Travaux.

LEMAN DELERIVE G. (1976). - "Le cimetière gaulois de Port-le-Grand (Somme). Essai d'interprétation des fouilles de 1833-1834». Cahiers Archéologiques de Picardie, 3, p. 97-113, X pl.

SIMON Ph. (1969). - "Céramiques protohistoriques découvertes dans les sablières de Sandrancourt (Val d'Oise)». Bulletin Archéologique du Vexin francais, 5, p. 49-51, 1 fig.

SIMON Ph. (1973). - «A propos des céramiques de La Tène I découvertes à Sandrancourt (Val d'Oise)». Bulletin Archéologique du Vexin francais, 9, p. 57-58, 1 fig. 